\title{
Dynamics of Large Debris Connected to Space Tug by a Tether
}

\author{
Vladimir S. Aslanov* and Vadim V. Yudintsevı̇ \\ Samara State Aerospace University, 443086 Samara, Russia
}

DOI: $\underline{10.2514 / 1.60976}$

\begin{abstract}
A spatial motion of a large passive satellite (space debris) and a space tug connected by an elastic tether is considered. The motion of the system is excited by a thrust force acting on the space tug. Major attention is given to a derivation of the equations of the motion based on the Lagrange formalism. Correctness of the mathematical model is proved by the theorem on variation of angular momentum and by the numerical simulations. The influence of the initial conditions and the system parameters on the behavior of the passive satellite is studied. The possibility of critical modes of the system motion leading to entanglement of the tether is shown by means of the numerical simulations.
\end{abstract}

\section{Nomenclature}

$A, B, C=$ moments of inertia of the passive satellite, $\mathrm{kg} \cdot \mathrm{m}^{2}$ $a, b, c=$ coordinates of the tether-attachment point in the coordinate frame $S x y z, \mathrm{~m}$

$c_{t}=$ tether stiffness, $\mathrm{N} / \mathrm{m}$

$\mathbf{K}_{H} \quad=$ angular-momentum vector of the system relative to the point $T, \mathrm{~kg} \cdot \mathrm{m}^{2} / \mathrm{s}$

$\mathbf{K}_{S} \quad=$ angular-momentum vector of the passive satellite relative to the point $S, \mathrm{~kg} \cdot \mathrm{m}^{2} / \mathrm{s}$

$k_{t}=$ tether-damping coefficient, $\mathrm{N} \cdot \mathrm{s} / \mathrm{m}$

$l \quad=\quad$ length of the tether, $\mathrm{m}$

$m_{H} \quad=\quad$ mass of the space tug, $\mathrm{kg}$

$m_{S} \quad=$ mass of the passive satellite (space debris), $\mathrm{kg}$

$p_{\psi}, p_{\varphi}=$ generalized impulses, $\mathrm{kg} \cdot \mathrm{m}^{2} / \mathrm{s}$

$s=$ distance between centers of mass of the space tug and the passive satellite, $\mathrm{m}$

Sxyz $=$ principal coordinate frame of the passive satellite

$S x_{c} y_{c} z_{c}=$ coordinate frame aligned to the line between the center mass of the passive satellite and the center mass of the space tug

$T=$ kinetic energy of the system, $J$

$\psi, \theta, \varphi=$ Euler angles that describe attitude motion of the passive satellite relative to the frame $S x_{c} y_{c} z_{c}$, rad

\section{Introduction}

$\mathbf{T}$ HE advent of tethered satellite systems (TSS) starts a new era in space research. The knowledge of dynamics and simulation of the tethered satellite systems during deployment and retrieval is essential for such a study [1-ㄷ]. TSS emerged as a new technology in space-related missions.

The density of debris in Earth's orbits is increasing dramatically. This fact reduces the availability of some orbits due to high risk of collision with the space debris. Moreover, the problem is compounded by every collision of the space debris, which creates more debris. Controlling the growth of the orbital-debris population is a high-priority task of the world community. There are several technical solutions being proposed for space-debris mitigation [611]. One of these uses a tethered removal satellite, a debritor [7], to

Received 29 October 2012; revision received 4 February 2013; accepted for publication 7 February 2013; published online XX epubMonth XXXX. Copyright $@ 2013$ by the American Institute of Aeronautics and Astronautics, Inc. All rights reserved. Copies of this paper may be made for personal or internal use, on condition that the copier pay the $\$ 10.00$ per-copy fee to the Copyright Clearance Center, Inc., 222 Rosewood Drive, Danvers, MA 01923; include the code 1533-3884/YY and \$10.00 in correspondence with the CCC.

*Head, Theoretical Mechanics Department, 34 Moskovskoe sh.; aslanov_vs@mail.ru.

${ }^{\dagger}$ Associate Professor, Theoretical Mechanics Department, 34 Moskovskoe sh.; yudintsev@classmech.ru. transport nonoperational debris objects from populated orbits. The debritor has to perform a rendezvous maneuver, attach a tether to the space debris, and deorbit the debris or change its orbit. This will minimize future risk to space objects orbiting in the same region. One of the motivating applications of this work is transporting space debris (nonfunctional satellites or upper stages of the rockets) from the orbit using a tethered space tug. Large space debris can strongly affect the motion of the debritor and the tether during the transportation, which can lead to the loss of control of the TSS. Therefore, the process of transportation must be analyzed in detail.

In this paper we consider space debris as a passive nonfunctional satellite or an upper stage of a rocket as a rigid body hereafter referred to as a passive satellite. The debritor (also referred to as active satellite or space tug) is considered as a mass point. The debritor is equipped with a rocket thruster and connected to the passive satellite by a viscous-elastic weightless tether. We investigate only the behavior of the passive satellite relative to the tether under the action of the tethertension force; therefore, the motion of the system is considered in space without gravity. The Lagrangian-formulation procedure is utilized to obtain the governing ordinary-differential equations of the spatial motion for the proposed system. The theorem on variation of angular momentum and results of numerical modeling are used to validate the equations of the motion. The set of exact governing equations of motion is numerically integrated to analyze the impact of system parameters and initial conditions to the motion of the system.

\section{Description of the System and Equations of Motion}

The system is comprised of the tether connecting the active satellite (space tug) with the passive satellite (Fig. 1). The tether is assumed to be made of a light but rigid material such as Kevlarfi and, hence, is taken to have negligible mass that is not considered in the calculations. The tether's transverse and torsional vibrations are ignored.

The axes of the coordinate frame $O x_{0} y_{0} z_{0}$ passing through the system center of mass $O$ are parallel to the axes of inertial reference frame. The origin of the coordinate frame $S x_{c} y_{c} z_{c}$ is at the mass center of the space debris $S$. The axis $S x_{c}$ is directed along the line $S H$ that joins the centers of mass of the space debris and the space tug. The axis $S z_{c}$ is directed in the opposite direction of the axis $O z_{0}$, and the axis $S y_{c}$ is defined to be parallel to the plane $O x_{0} y_{0}$, and it completes the right-hand triad. The frame $S x y z$ is the coordinate frame fixed at the space debris. The axes $S x, S y, S z$ are the principal central axes of inertia of the space debris. The orientation of the coordinate frame $S x y z$ relative to the coordinate frame $S x_{c} y_{c} z_{c}$ is specified by a set of three successive Euler rotations (precession $\psi$, nutation $\theta$, and $\operatorname{spin} \varphi$ ) [12]. We assume the centerline $S H$ remains in the horizontal plane $O x y$ and the thrust of the debritor $\mathbf{F}$ acts only in this plane during the motion of the system. 


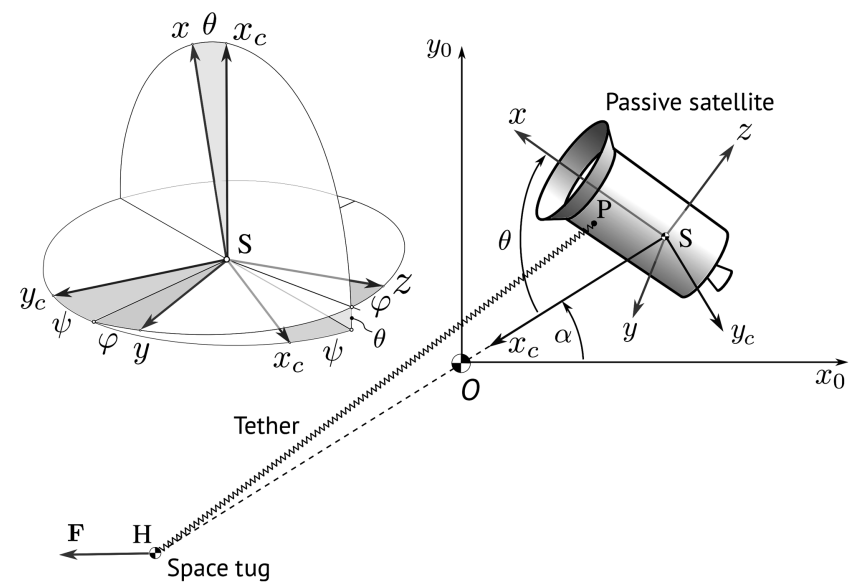

Fig. 1 Space debris and space tug connected by the tether.

To obtain the equations of motion the most appropriate approach seems to be the Lagrangian technique. Lagrange's equations present an energy method of dynamic analysis based on finding expressions for the kinetic energy $T$ of the system, and as well, its generalized forces $Q_{i}$. Lagrange's equations have the following general form [12]

$$
\frac{\mathrm{d}}{\mathrm{d} t} \frac{\partial T}{\partial \dot{q}_{i}}-\frac{\partial T}{\partial q_{i}}=Q_{i}, \quad i=1,2 \ldots, 7
$$

where $q_{i},(i=1,2, \ldots, 7)$ are the generalized coordinates, $q_{1,2} \equiv$ $x, y$ are coordinates of the center of mass of the system in the inertial coordinate system, $q_{3} \equiv S H=s, q_{4} \equiv \alpha$ is an angle between the line $S H$ and the axis $O x_{o}, q_{5} \equiv \psi, q_{6} \equiv \theta$, and $q_{7} \equiv \varphi$ are Euler angles, and $Q_{i}$ is a generalized force corresponding to the generalized coordinate $q_{i}$. The tether length $l=P H$ is written as

$$
l=\sqrt{\rho^{2}-2 s[a \cos \theta+(b \sin \varphi+c \cos \varphi) \sin \theta]+s^{2}}
$$

where $\rho=S P=\sqrt{a^{2}+b^{2}+c^{2}}$, and $a, b, c$ are the coordinates of the point $P$ where the tether is attached at the passive satellite.

The system kinetic energy $T$ can then be written as

$$
\begin{aligned}
T & =\frac{m_{H}+m_{S}}{2}\left(\dot{x}^{2}+\dot{y}^{2}\right)+\frac{m_{H} m_{S}}{2\left(m_{H}+m_{S}\right)}\left(\dot{s}^{2}+s^{2} \dot{\alpha}^{2}\right) \\
& +\frac{1}{2}\left\{A[\dot{\alpha} \sin \theta \cos \psi+\dot{\psi} \cos \theta+\dot{\varphi}]^{2}\right. \\
& +B[\dot{\alpha}(\cos \theta \sin \varphi \cos \psi+\cos \varphi \sin \psi) \\
& +\dot{\psi} \sin \theta \sin \varphi+\dot{\theta} \cos \varphi]^{2} \\
& +C[\dot{\alpha}(\cos \theta \cos \varphi \cos \psi-\sin \varphi \sin \psi) \\
& \left.+\dot{\psi} \sin \theta \cos \varphi-\dot{\theta} \sin \varphi]^{2}\right\}
\end{aligned}
$$

where $m_{H}$ and $m_{S}$ are masses of the active satellite and the passive satellite, respectively, and $(A, B, C)$ are central principal moments of inertia of the passive satellite.

The generalized forces $Q_{i}$ are determined by the following formulas

$$
\begin{aligned}
Q_{x}= & F_{x}, \quad Q_{y}=F_{y}, \\
Q_{\alpha} & =\frac{m_{S} s}{m_{H}+m_{S}}\left(F_{x} \sin \alpha-F_{y} \cos \alpha\right), \\
Q_{s} & =-\frac{m_{S}}{m_{H}+m_{S}}\left(F_{x} \cos \alpha+F_{y} \sin \alpha\right) \\
+ & \xi[a \cos \theta+(b \sin \varphi+c \cos \varphi) \sin \theta-s] \\
& \times\left(c_{t} \frac{l-l_{0}}{l}+k_{t} l\right), \quad Q_{\psi}=0, \\
Q_{\theta} & =\xi_{s}[(b \sin \varphi+c \cos \varphi) \cos \theta-a \sin \theta]\left(c_{t} \frac{l-l_{0}}{l}+k_{t} i\right), \\
Q_{\varphi} & =\xi_{s} \sin \theta(b \cos \varphi-c \sin \varphi)\left(c_{t} \frac{l-l_{0}}{l}+k_{t} i\right)
\end{aligned}
$$

where $c_{t}$ is tether stiffness, $k_{t}$ is tether damping, and $\xi$ is a unit step function

$$
\xi=\left\{\begin{array}{l}
1, \quad l-l_{0} \geq 0 \\
0, \quad l-l_{0}<0
\end{array}\right.
$$

Substituting the kinetic energy [Eq. (3)] and the generalized forces [Eq. (4)] in the Lagrange equations [Eq. (1)] and carrying out the differentiation we can get the governing nonlinear coupled ordinarydifferential equations of the spatial motion of the system. However, these equations are very cumbersome and can be used only for the numerical simulation. Next, we write a simplified version of these equations.

\section{Equations of the Spatial Motion of the Axisymmetric Passive Satellite Relative to the Tether}

Let us write the equations of the spatial motion of the system with these assumptions: the passive satellite has an axisymmetric inertia tensor $(A, B=C)$, the tether is attached at the axis of symmetry $(a \neq 0, b=c=0)$, and the tether damping is absent $\left(k_{t}=0\right)$. In this case kinetic energy [Eq. (3)] of the system has the form

$$
\begin{aligned}
T & =\frac{1}{2}\left[A(\dot{\varphi}+\dot{\psi} \cos \theta)^{2}+B\left(\dot{\theta}^{2}+\dot{\psi}^{2} \sin ^{2} \theta\right)+\frac{m_{H} m_{S}}{m_{H}+m_{S}} \dot{s}^{2}\right. \\
& \left.+\left(m_{1}+m_{2}\right)\left(\dot{x}^{2}+\dot{y}^{2}\right)\right]
\end{aligned}
$$

We also suppose that the tether length $l$ is much greater than $S P=a$

$$
l \gg a
$$

Then the tether length can be expressed as

$$
l \approx s-a \cos \theta
$$

At the initial time $\alpha=0$ and the space-tug thrust force acts along the $x$-axis $F_{x}=F, F_{y}=F_{z}=0$. In this case generalized forces [Eq. (4)] are expressed as

$$
\begin{gathered}
Q_{x}=F, \quad Q_{y}=0, \quad Q_{\alpha}=0, \quad Q_{\psi}=0, \quad Q_{\varphi}=0 \\
Q_{s}=-\frac{m_{S}}{m_{H}+m_{S}} F+\xi c_{t} l, \quad Q_{\theta}=-\xi c_{t} a l \sin \theta
\end{gathered}
$$

Here we consider the spatial motion of the passive satellite relative to the tether only, and the differential equations of the center of mass of the system are omitted. Note that the kinetic energy [Eq. (5)] doesn't depend on the coordinates $\psi$ and $\varphi$, and we can write two first integrals [12] 


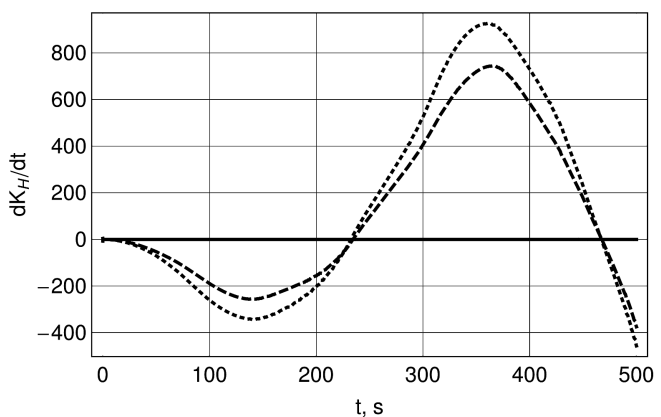

a)

Fig. 2 Components of the left-hand side and right-hand side of Eq. (12). Projection on the axes $x_{0}, y_{0}$, and $z_{0}$ depicted as solid, dashed, and dotted lines, respectively.

$$
\begin{aligned}
& p_{\varphi}=\frac{\partial T}{\partial \dot{\varphi}}=A(\dot{\varphi}+\dot{\psi} \cos \theta)=\mathrm{const} \\
& p_{\psi}=\frac{\partial T}{\partial \dot{\psi}}=A \dot{\varphi} \cos \theta+\dot{\psi}\left(A \cos ^{2} \theta+B \sin ^{2} \theta\right)=\mathrm{const}
\end{aligned}
$$

Taking into account these first integrals the equations of the spatial motion of the passive satellite have the simple form

$$
\begin{aligned}
& \ddot{s}+k_{t} \frac{m_{H}+m_{S}}{m_{H} m_{S}} s=c_{t} \frac{m_{H}+m_{S}}{m_{H} m_{S}}\left(a \cos \theta+l_{0}\right)-\frac{m_{S}}{m_{H}} \mathrm{~F} \\
& \ddot{\theta}+\frac{(R-G \cos \theta)(R-G \cos \theta)}{\sin ^{3} \theta}+\frac{a c_{t}}{B} s \sin \theta=\frac{a^{2} c_{t}}{2 B} \sin 2 \theta
\end{aligned}
$$

where $R=p_{\varphi} / B, G=p_{\psi} / B$. So far as Euler angles are used to describe attitude motion of the passive satellite; Eq. (11) is correct while $\sin \theta \neq 0$.

We note that Eq. (11) has a lot of similarity to the equation of the Lagrange case [13]. The tether force acts like a gravity force, but here the tether force is a function of time. There is a small periodic term at the right side of Eq. (11) according to the assumption [Eq. (6)]. Furthermore, Eq. (11) is similar to the equations of the spatial motion of the axisymmetric spacecraft in the atmosphere [14].

\section{Validation of the Mathematical Model}

To validate the correctness of the equations let us write a variation of the angular-momentum vector $\mathbf{K}_{H}$ relative to the moving point $H$ [15]

$$
\begin{aligned}
\frac{\mathrm{d} \mathbf{K}_{H}}{\mathrm{~d} t} & =\left(m_{H}+m_{S}\right) \mathbf{V}_{O} \times \mathbf{V}_{H}+M_{H} \\
& =\left(\mathbf{V}_{H} m_{H}+\mathbf{V}_{S} m_{S}\right) \times \mathbf{V}_{H}+M_{H}=m_{S} \mathbf{V}_{S} \times \mathbf{V}_{H}
\end{aligned}
$$

In Eq. (12) $M_{H}=0$ because of the tether attached at point $H$, and the moment of the tether force relative to point $H$ equals zero. In the inertial frame the angular-momentum vector is defined as [1]

$$
\begin{aligned}
\mathbf{K}_{H}= & \mathbf{J}_{S} \boldsymbol{\omega}_{S}+\mathbf{R}_{S} \times\left(m_{S} \mathbf{V}_{S}\right)+\mathbf{R}_{H} \times\left(m_{H} \mathbf{V}_{H}\right)+\mathbf{R}_{H} \\
& \times\left[\left(m_{H}+m_{S}\right) \mathbf{V}_{0}\right]
\end{aligned}
$$

$\mathbf{V}_{S}$ is a velocity of the passive satellite relative to the inertial frame

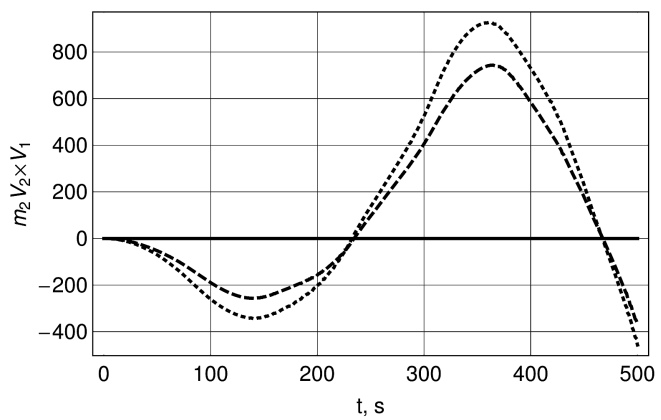

b)

$$
\mathbf{V}_{S}=\mathbf{V}_{0}+\frac{\mathrm{d} \mathbf{R}_{S}}{\mathrm{~d} t}, \quad \mathbf{R}_{S}=\mathbf{O S}=\frac{m_{H} s}{m_{H}+m_{S}}\left(\begin{array}{c}
\cos \alpha \\
\sin \alpha \\
0
\end{array}\right)
$$

where $\mathbf{V}_{H}$ is a velocity vector of the space tug

$$
\mathbf{V}_{H}=\mathbf{V}_{0}+\frac{\mathrm{d} \mathbf{R}_{H}}{\mathrm{~d} t}, \quad \mathbf{R}_{H}=\mathbf{O H}=-\frac{m_{S} s}{m_{H}+m_{S}}\left(\begin{array}{c}
\cos \alpha \\
\sin \alpha \\
0
\end{array}\right)
$$

where $\mathbf{V}_{0}$ is a velocity vector of the system center of mass.

The absolute angular velocity of the passive satellite is

where $\boldsymbol{\Omega}_{S}$ is an angular velocity of the passive satellite relative to the frame $S x_{c} y_{c} z_{c}$

$$
\boldsymbol{\Omega}_{S}=\left(\begin{array}{l}
\dot{\varphi}+\dot{\psi} \cos \theta \\
\dot{\theta} \cos \varphi+\dot{\psi} \sin \theta \sin \varphi \\
-\dot{\theta} \sin \varphi+\dot{\psi} \sin \theta \cos \varphi
\end{array}\right)
$$

The angular velocity of the frame $S x_{c} y_{c} z_{c}$ relative to the inertial frame is

$$
\omega_{c}=\mathbf{k}_{c} \dot{\alpha}
$$

where $\mathbf{k}_{c}$ is a unit vector of the axis $S x_{c}$.

After obtaining the solution of the differential equations [Eq. (1)] of the system we can substitute the solution in to Eqs. (13)-(15) and build Eq. (12). If the right-hand side and the left-hand side of Eq. (12) are the same, the solution is correct. In Fig. 2 the components of the left-hand-side vector and the right-hand-side vector of Eq. (12) are shown. The relative numerical integration error $\varepsilon_{K}$ is

$$
\varepsilon_{K}=\frac{\left|\dot{\mathbf{K}}_{H}-m \mathbf{V}_{0} \times \mathbf{V}_{H}\right|}{\left|\dot{\mathbf{K}}_{H}\right|}<10^{-4}
$$

Table 1 presents the result for the parameters of the system and the following initial conditions

$$
\boldsymbol{\omega}_{S}=\boldsymbol{\omega}_{c}+\boldsymbol{\Omega}_{T}
$$

Table 1 Parameters of the system.

\begin{tabular}{lccccccc}
\hline \hline Parameter & Value & Parameter & Value & Parameter & Value & Parameter & Value \\
\hline$A, \mathrm{~kg} \cdot \mathrm{m}^{2}$ & 3000 & $F_{x}, \mathrm{~N}$ & -10 & $a, \mathrm{~m}$ & 2.5 & $m_{1}, \mathrm{~kg}$ & 1000 \\
$B, \mathrm{~kg} \cdot \mathrm{m}^{2}$ & 10,000 & $F_{y}, \mathrm{~N}$ & 0 & $b, \mathrm{~m}$ & 0 & $m_{2}, \mathrm{~kg}$ & 3000 \\
$C, \mathrm{~kg} \cdot \mathrm{m}^{2}$ & 10,000 & $F_{z}, \mathrm{~N}$ & 0 & $c, \mathrm{~m}$ & 0 & $l_{0}, \mathrm{~m}$ & 50 \\
\hline \hline
\end{tabular}




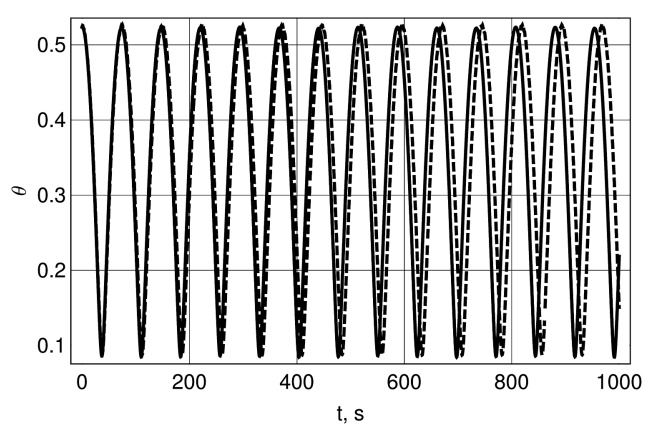

a)

$$
\begin{aligned}
& \varphi_{0}=\psi_{0}=\dot{s}_{0}=\dot{\theta}_{0}=0 \quad \theta_{0}=\pi / 3, \\
& \dot{\varphi}_{0}=0.05 \mathrm{rad} / \mathrm{s} \quad s_{0}=s_{*}
\end{aligned}
$$

where $s_{*}$ is the solution of Eq. (2) for $l=l_{0}$.

In the Fig. 3 the solutions for angle $\theta$ of the accurate differential equations [Eq. (1)] and the approximate equations [Eqs. (10) and (11)] are shown for two lengths of the tether. We note that approximate
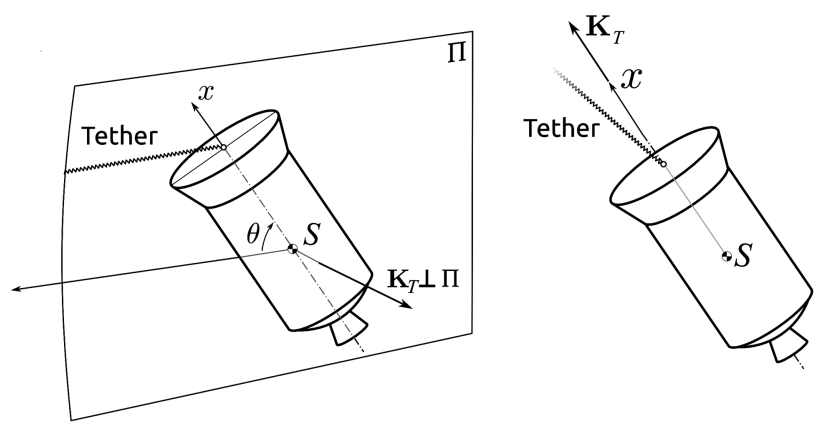

a)
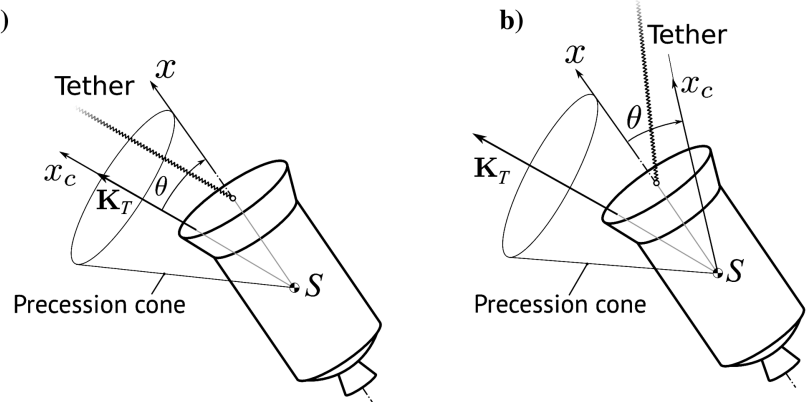

c)

d)

Fig. 4 Initial orientations of the passive satellite relative to the angularmomentum vector and relative to the tether.

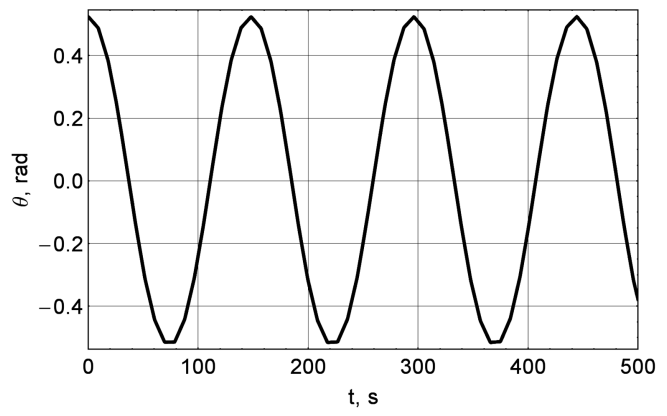

Fig. 5 Time history of angle $\theta$ for case 1 when $l(0)-l_{0}=0$.

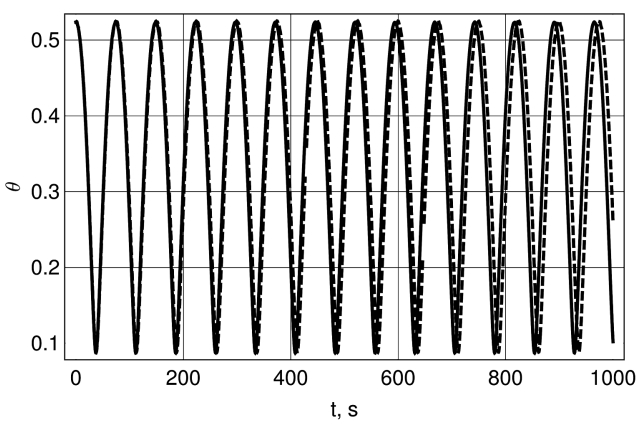

b)

solution of Eqs. (10) and (11) close to the solution of the accurate differential equations for the longer tether.

\section{Numerical Results}

Here we investigate the behavior of the passive satellite depending on its initial position relative to the tether. We consider four types of the initial orientation of the passive satellite relative to the tether and

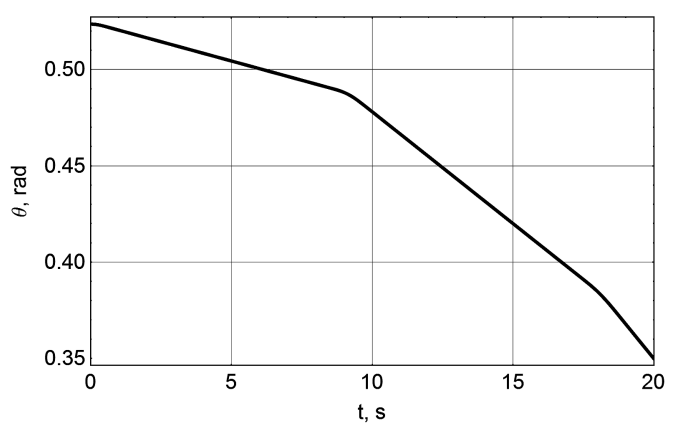

Fig. 6 Time history of angle $\theta$ for case 1 when $l(0)-l_{0}=0$ and time interval $[0 ; 20 \mathrm{~s}]$.

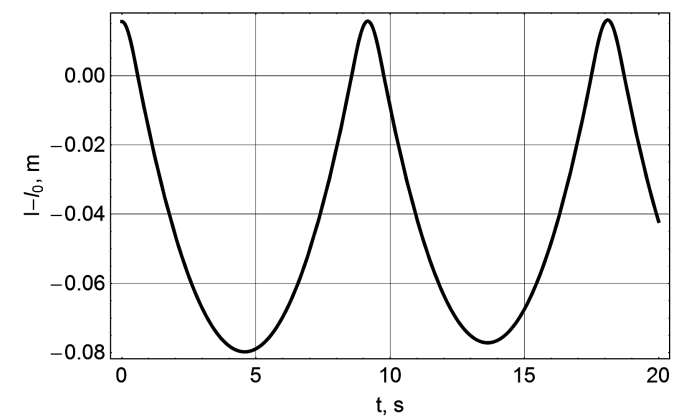

Fig. 7 Time history of tether elongation $l-l_{o}$ for case 1 when $l(0)-$ $l_{0}=0$ and time interval $[0 ; 20 \mathrm{~s}]$.

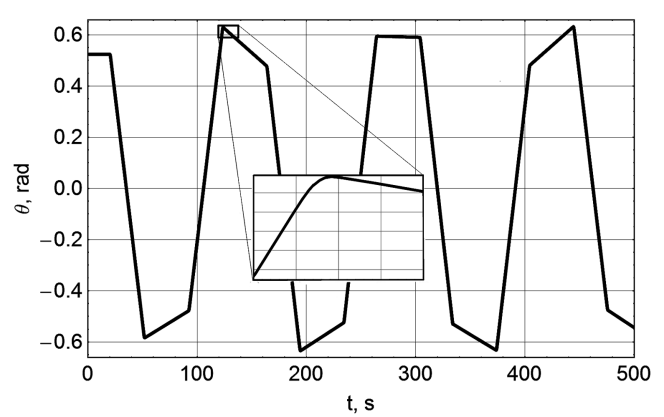

Fig. 8 Time history of angle $\theta$ for case 1 when $l(0)-l_{0}=-2 \mathrm{~m}$. 


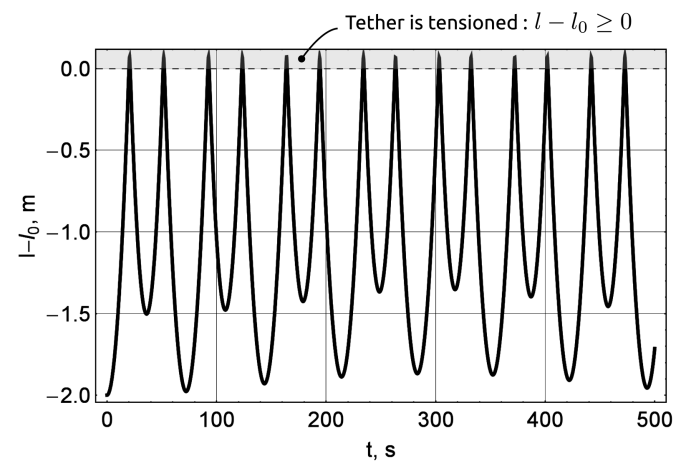

Fig. 9 Time history of tether elongation $l-l_{o}$ for case 1 when tether is slack at $t=0$.

the initial angular-momentum vector $\mathbf{K}_{S}$ (Fig. 4): 1) case 1: $\mathbf{K}_{S} \perp S x$, $\mathbf{K}_{S} \perp S x_{c}, \dot{\varphi}_{0}=0$ (Fig. 4a) $; 2$ ) case $2:\left(\mathbf{K}_{S}\right)_{t=0} \| S x$ (Fig. $\left.\underline{4 b}\right) ; 3$ ) case 3: $\left(\mathbf{K}_{S}\right)_{t=0} \| S x_{c}$ (Fig. 4c); 4) case 4, a general case, in which vectors $\mathbf{K}_{S}, S x, S x_{c}, \mathbf{R}_{12}$ are arbitrarily oriented in space (Fig. 4d).

Motion of the passive satellite is investigated for the tense and slack tether at the initial time for the four cases. Parameters of the system and initial conditions are presented in the Table 1 and in Eq. (17).

\section{A. Case 1}

At first we consider the plane motion of the passive satellite when its angular-momentum vector remains perpendicular to the axis of symmetry $S x$ (Fig. 4a). Figure $\underline{5}$ shows the time history of angle $\theta$ when $\dot{\psi}_{0}=0, \dot{\varphi}_{0}=0, \dot{\theta}_{0}=0$, and the tether is tensioned at $t=0$. Figures $\underline{6}$ and 7 show time histories of angle $\theta$ and the tether elongation for time interval from 0 to $20 \mathrm{~s}$. These two figures explain the discontinuity in slope of angle $\theta$ in Fig. $\underline{5}$. When the tether slacks the slope of the function $\theta$ changes.
If the motion starts when $l(0)=l_{o}-\delta_{l}\left(\delta_{l}=2 \mathrm{~m}\right.$, slack tether $)$ the amplitude of angle $\theta$ increases (Fig. 8). The tether acts on the passive satellite at short time intervals that lead to the higher amplitude of angle $\theta$. Most of the time the tether is slack (Fig. 9). Angle $\theta$ changes linearly in time while tether is slack (passive spacecraft rotates with constant angular velocity). In the short time intervals the tether becomes tensioned and quickly changes the angular velocity of the passive spacecraft (compare Figs. 8 and 9). This type of transportation is objectionable due to risk of tether rupture.

\section{B. Case 2}

Next we consider a case when the angular-velocity vector of the passive satellite parallel to the axis of symmetry of the passive satellite (Fig. 4b). The initial angular velocity of the passive satellite is $\left|\omega_{2}\right|=\dot{\varphi}_{0}=\overline{0.05} \mathrm{rad} / \mathrm{s}$. Figure 10a shows the time history of angle $\theta$. As with in the previous case with tensioned tether the oscillations of angle $\theta$ are such that $\left|\max _{t}(\theta)\right|<\left|\theta_{0}\right|$. The evolution of angle $\theta$ for the slack tether $\left[l(0)=l_{o}-\delta_{l}\right]$ is shown in Fig. $\underline{10 \mathrm{~b}}$.

\section{Case 3}

Let us consider the motion of the passive satellite when the initial angular-momentum vector is parallel to the axis $S x_{c}$ (Fig. 4c). The system starts its motion with the initial conditions [Eq. (17)] and

$$
\dot{\varphi}_{0}=0.05 \frac{\mathrm{rad}}{\mathrm{s}}, \quad \dot{\psi}_{0}=0.0247 \frac{\mathrm{rad}}{\mathrm{s}}
$$

for which $\mathbf{K}_{S}$ is parallel to the axis $S x_{c}$. Figures $11 \mathrm{a}$ and $11 \mathrm{~b}$ show the time histories of angle $\theta$ for the tension tether and the slack tether at $t=0$.

\section{Case 4}

Here we consider the behavior of the passive satellite with initial conditions [Eq. (17)] and

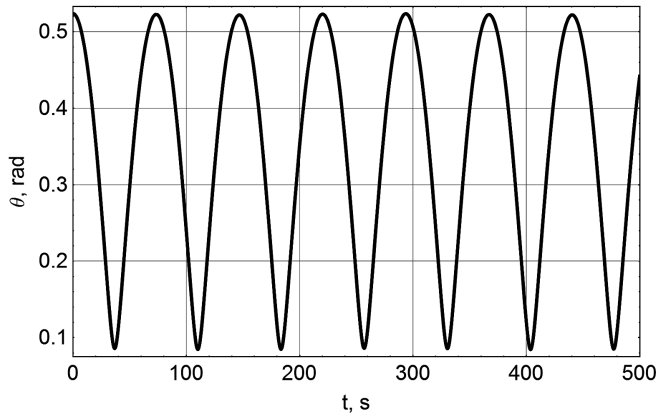

a)

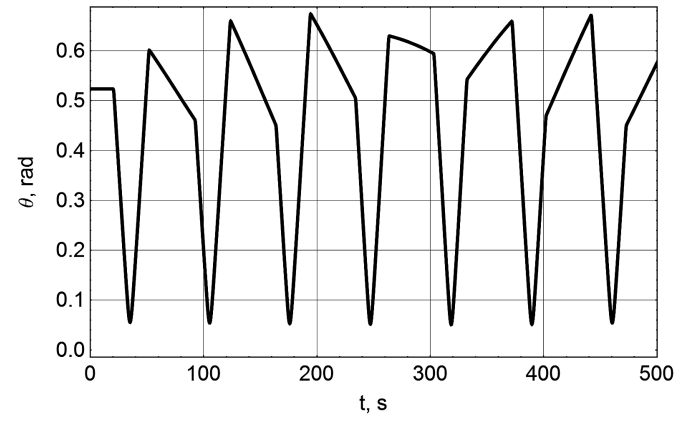

b)

Fig. 10 Time history of angle $\theta$ for case 2.

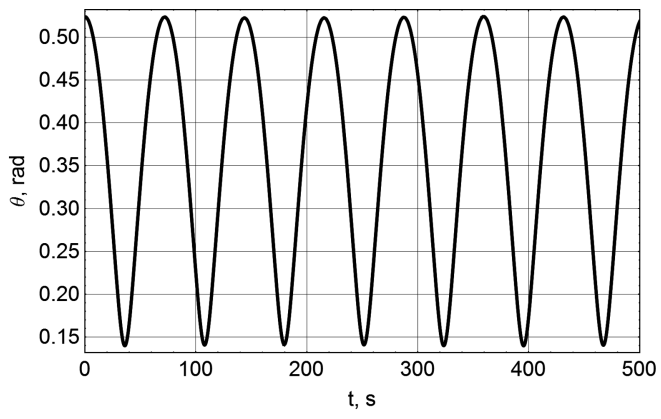

a)

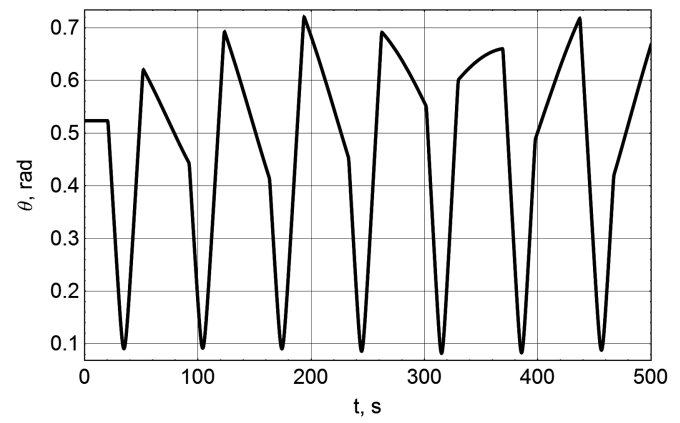

b)

Fig. 11 Time history of angle $\theta$ for case 3. 


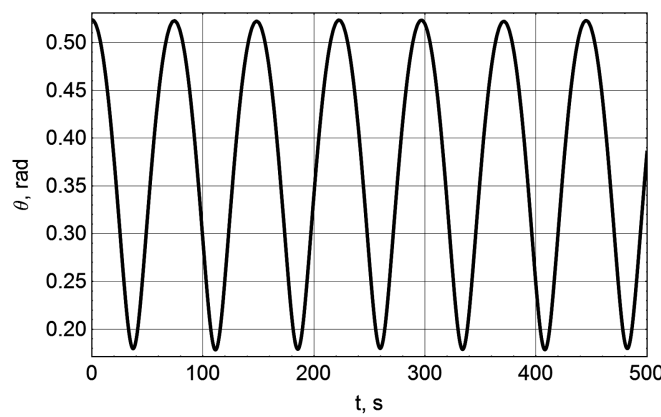

a)

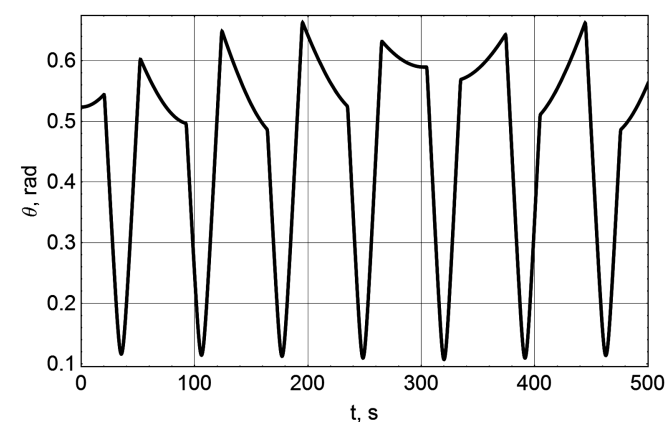

b)

Fig. 12 Time history of angle $\theta$ for case 4.

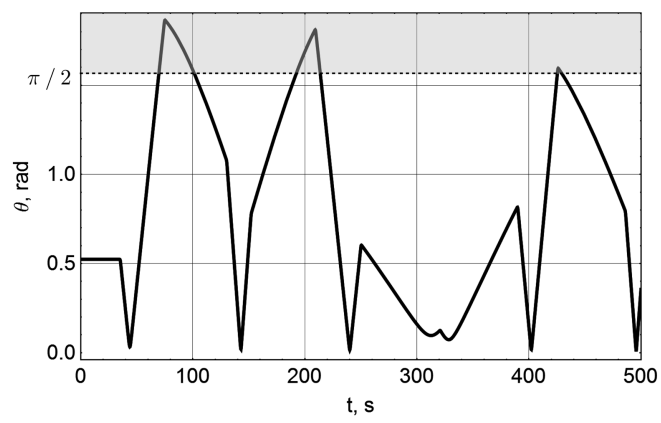

a)

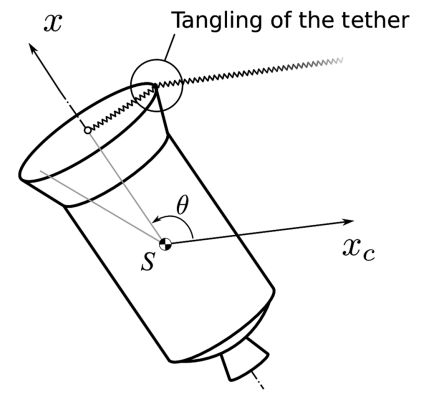

b)

Fig. 13 Time history of angle $\theta$ for large initial slackness of the tether and possible orientation of the passive satellite that can lead to tether rupture.

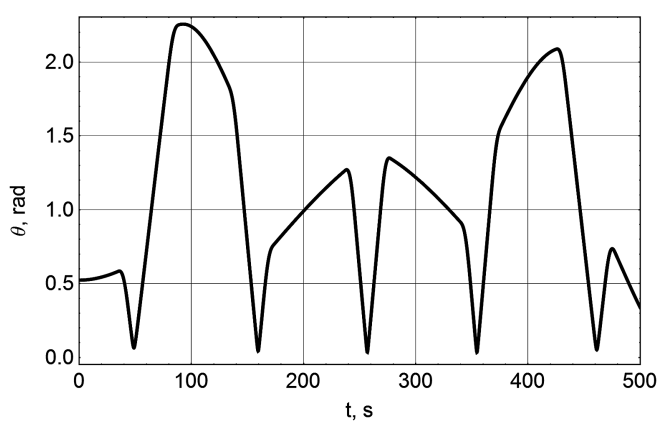

Fig. 14 Time history of angle $\theta$ for case $4\left(c_{t}=1 \mathrm{GPa}, \delta_{l}=6 \mathrm{~m}\right)$.

$$
\dot{\varphi}_{0}=0.05 \frac{\mathrm{rad}}{\mathrm{s}}, \quad \dot{\psi}_{0}=0.01 \frac{\mathrm{rad}}{\mathrm{s}}
$$

In this case the tether passes from the outside of the precession cone

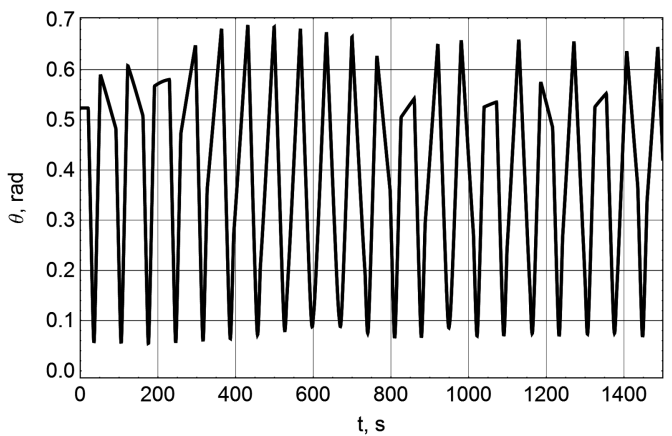

a)

$$
\frac{K_{S x}}{\left|\mathbf{K}_{S}\right|}<\cos \theta
$$

Figure 12 shows the time histories of angle $\theta$ for the tension tether and the slack tether at $t=0$.

We haven't noticed any significant difference in the behavior of the passive satellite in any particular case. If we take more slackness of the tether, e.g., $\delta_{l}=6 \mathrm{~m}$, the high amplitude of $\theta$ can result in the tether contacting the passive satellite. The tether can tangle or break. Figure 13 shows the case when angle $\theta$ exceeds the value $\pi / 2$.

We can expect a higher amplitude of angle $\theta$ for the softer tether. Figure 14 shows the time history of angle $\theta$ for $\delta_{l}=6 \mathrm{~m}$ and $c_{t}=1 \overline{\mathrm{GPa}}$.

Next we investigate the motion of the system with a damped tether. Tether damping can decrease its amplitude of oscillations. Figure 15a shows the time history of angle $\theta$ for the system with initial conditions [Eq. (17)] and the damping coefficient $k_{d}=10 \mathrm{Ns} / \mathrm{m}$. Figure $15 \mathrm{~b}$ shows the time history of the tether elongation. We note that the damping properties of the tether affect high oscillations of the tether, but it doesn't affect the oscillation of angle $\theta$.

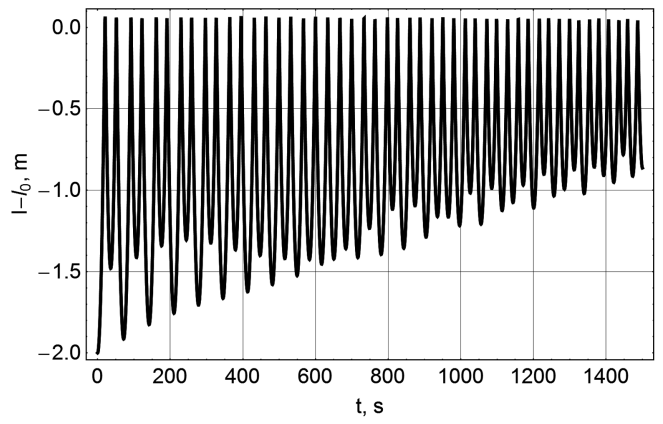

b)

Fig. 15 Time history of angle $\theta$ and the elongation of the tether with damping. 


\section{Conclusions}

The motion equations of the space-tether system are built upon the Lagrange formalism. The simplified equations of the spatial motion of the passive satellite relative to the tether are developed. The correctness of the equations is validated by means of the angularmomentum-variation law. The possibility of transporting the passive satellite is shown. The spatial motion of the passive satellite is considered for the different cases of the system's parameters. It is shown that the amplitude of oscillation of the passive satellite primarily depends on the properties of the tether. Slackness of the tether can lead to tether tangling due to the high amplitude of the oscillation of the passive satellite relative to the tether. For the safe transportation of the passive spacecraft the slackness of the tether and the high initial angle between the tether and the line SB that connects the center mass of the passive spacecraft with the tether-attachment point should be avoided.

\section{Acknowledgment}

This research was supported by Russian Foundation for Basic Research (12-01-00317-a).

\section{References}

[1] Troger, H., Alpatov, A. P., Beletsky, V. V., Dranovskii, V. I., Khoroshilov, V. S., and Pirozhenko, A. V., Dynamics of Tethered Space Systems, CRC Press, Boca Raton, FL, 2010.

[2] Beletsky, V., and Levin, E., Dynamics of Space Tether Systems, Advances in the Astronautical Sciences, Vol. 83, Univelt, Inc., San Diego, CA, 1993.

[3] Aslanov, V. S., and Ledkov, A. S., Dynamics of the Tethered Satellite Systems, Woodhead Publishing Ltd., Cambridge, England, U. K., 2012.

[4] Aslanov, V. S., "Orbital Oscillations of an Elastic Vertically-Tethered Satellite," Mechanics of Solids, Vol. 64, No. 5, 2011, pp. 657-668. doi: $10.3103 / \mathrm{S} 0025654411050013$
[5] Kumar, K. D., and Kumar, K., "Attitude Maneuver of Dual Tethered Satellite Platforms Through Tether Offset Change," Journal of Spacecraft and Rockets, Vol. 38, No. 2, 2001, pp. 237-242. doi: $10.2514 / 2.3676$

[6] Mankala, K. K., and Agrawal, S. K., "Dynamic Modeling and Simulation of Impact in Tether Net/Gripper Systems," Multibody System Dynamics, Vol. 11, No. 5, 2004, pp. 235-250. doi:10.1023/B:MUBO.0000029393.25494.64

[7] Cougnet, C., Alary, D., Gerber, B., Utzmann, J., and Wagner, A., "The Debritor: an "Off the Shelf" Based Multimission Vehicle," Proceedings of the 63rd International Astronautical Congress, Naples, Italy, 2012.

[8] Kaiser, C., Bellido, E., and Hofmann, P., "Space Debris Mitigation Using On-Orbit Servicing Solutions," Proceedings of the 63rd International Astronautical Congress, Naples, Italy, 2012.

[9] DeLuca, L. T., "Active Space Debris Removal by Hybrid Engine Module," Proceedings of the 63rd International Astronautical Congress, Naples, Italy, 2012.

[10] Sinn, T., Thakore, T., and Maier, P., "Space Debris Removal Using SelfInflating Adaptive Membrane," Proceedings of the 63rd International Astronautical Congress, Naples, Italy, 2012.

[11] Lee, E. Z. J., Seubert, C. R., Schaub, H., Trushlyakov, V., and Yutkin, V., "Tethered Tug For Large Low Earth Orbit Debris Removal," American Astronautical Society/AIAA Astrodynamics Specialists Conference, Charleston, SC, 2012.

[12] Schaub, H., and Junkins, J. L., Analytical Mechanics of Aerospace Systems, AIAA Education Series, AIAA, Reston, VA, 2003.

[13] Wittenburg, J., Dynamics of Multibody Systems, Springer-Verlag, Berlin, 2008.

[14] Aslanov, V. S., "Spatial Chaotic Vibrations When There is a Periodic Change in the Position of the Centre of Mass of a Body in the Atmosphere," Journal of Applied Mathematics and Mechanics, Vol. 73, 2009, pp. 179-187. doi:10.1016/i.jappmathmech.2009.04.007

[15] Levi-Civita, T., and Amaldi, U., "Lezioni di Meccanica Razionale: Pt. 1, 2 Dinamica del Sistemi con un Numero Finito di Gradi di Liberta," Lezioni di Meccanica Razionale, 1953. 


\section{Queries}

1. AU: Please confirm the six-digit postal code to the left of the city in the affiliation line is correct.

2. AU: Please check that the copyright statement is correct.

3. AU: Please spell out the definition for the acronym SB in the Conclusions.

4. AU: Please provide page range for Refs. $[1-3,12,13]$.

5. AU: If Refs. [7-11] are published proceedings, please provide the name and location of the publisher (NOT of the conference host) and the page range. If it is a conference paper, please provide the paper number and the organizer's name.

6. AU: Please provide volume, issue, page range and DOI number for Ref. [15]. 